\title{
Acupuncture and moxibustion for chronic fatigue syndrome in traditional Chinese medicine: a systematic review and meta- analysis
}

Taiwu Wang, Cong Xu, Keli Pan and Hongyan Xiong ${ }^{*}$

\begin{abstract}
Background: As the etiology of chronic fatigue syndrome (CFS) is unclear and the treatment is still a big issue. There exists a wide range of literature about acupuncture and moxibustion (AM) for CFS in traditional Chinese medicine (TCM). But there are certain doubts as well in the effectiveness of its treatment due to the lack of a comprehensive and evidence-based medical proof to dispel the misgivings. Current study evaluated systematically the effectiveness of acupuncture and moxibustion treatments on CFS, and clarified the difference among them and Chinese herbal medicine, western medicine and sham-acupuncture.

Methods: We comprehensively reviewed literature including PubMed, EMBASE, Cochrane library, CBM (Chinese Biomedical Literature Database) and CNKI (China National Knowledge Infrastructure) up to May 2016, for RCT clinical research on CFS treated by acupuncture and moxibustion. Traditional direct meta-analysis was adopted to analyze the difference between AM and other treatments. Analysis was performed based on the treatment in experiment and control groups. Network meta-analysis was adopted to make comprehensive comparisons between any two kinds of treatments. The primary outcome was total effective rate, while relative risks (RR) and 95\% confidence intervals $(\mathrm{Cl})$ were used as the final pooled statistics.

Results: A total of 31 randomized controlled trials (RCTs) were enrolled in analyses. In traditional direct metaanalysis, we found that in comparison to Chinese herbal medicine, CbAM (combined acupuncture and moxibustion, which meant two or more types of acupuncture and moxibustion were adopted) had a higher total effective rate (RR (95\% Cl), $1.17(1.09 \sim 1.25)$ ). Compared with Chinese herbal medicine, western medicine and sham-acupuncture, SAM (single acupuncture or single moxibustion) had a higher total effective rate, with RR ( $95 \% \mathrm{Cl}$ ) of 1.22 (1.14 1.30), 1.51 (1.31-1.74), 5.90 (3.64-9.56). In addition, compared with SAM, CbAM had a higher total effective rate (RR (95\% Cl), $1.23(1.12 \sim 1.36))$. In network meta-analyses, similar results were recorded. Subsequently, we ranked all treatments from high to low effective rate and the order was CbAM, SAM, Chinese herbal medicine, western medicine and sham-acupuncture.
\end{abstract}

Conclusions: In the treatment of CFS, CbAM and SAM may have better effect than other treatments. However, the included trials have relatively poor quality, hence high quality studies are needed to confirm our finding.

Keywords: Acupuncture and moxibustion, Chronic fatigue syndrome, Traditional meta-analysis, Network meta-analysis, Acupoint

\footnotetext{
*Correspondence: hongyanxiong@126.com

Department of Epidemiology, College of Preventive Medicine, Third Military

Medical University, Gaotanyan Road 30, Shapingba District, Chongqing

400038, China
} International License (http://creativecommons.org/licenses/by/4.0/), which permits unrestricted use, distribution, and reproduction in any medium, provided you give appropriate credit to the original author(s) and the source, provide a link to the Creative Commons license, and indicate if changes were made. The Creative Commons Public Domain Dedication waiver (http://creativecommons.org/publicdomain/zero/1.0/) applies to the data made available in this article, unless otherwise stated. 


\section{Background}

CFS is a wide-spread agnogenic disease [1] characterized by unexplained fatigue that lasts for at least six months and accompanied by four or more of the following symptoms: unrefreshing sleep, lengthy malaise after exertion, impairment of concentration or short-term memory, sore throat, tender lymph nodes, multi-joint and muscle pain and headaches [2]. CFS affects work and life seriously, at the same time causes heavy societal burden with an incidence of $0.2 \% \sim 2.2 \%$ in adults [3, 4], $0.1 \% \sim 0.5 \%$ in adolescents [5] in western countries and $1.9 \%$ in Beijing and 3\% in Hong Kong, China [6]. American CDC reported a higher prevalence, with 2.3\% in children and $2.3 \%$ in adolescents (http://www.cdc.gov/ cfs/pediatric/index.html). Though many theories are used to explain CFS, the aetiology is still unclear and no major progress has been made in therapy. So far, in treatment of CFS, cognitive behavior [7-9] and graded exercise therapies $[10,11]$ are thought to be effective in relief of symptoms, and the curative effect of western medicines isn't promising. For instance, the effects of anxiolytics, corticosteroids, dietary supplements, evening primrose oil, homeopathy, magnesium (intramuscular), nicotinamide adenine dinucleotide (oral) and prolonged rest are unclear [12]. Suggestions on treatment have been made in a number of countries [13, 14], but their effect still needs to be further studied [15].

CFS has no clear mechanism, with its complex symptoms. As the treatment effectiveness by western medicine were limited overall, some researchers also begun to shift their focus towards complementary and alternative medicine. Acupuncture and moxibustion in traditional Chinese medicine (TCM) [16] comes to the attention of researchers. Acupuncture means the insertion of needles into different parts or points of the body and moxibustion mans the use of burning moxa to stimulate certain parts or points of the body, while moxa is usually made from a special herb named argy wormwood leaf. As the development of acupuncture and moxibustion, acupuncture and moxibustion have many different forms, for example, both auricular acupuncture and electroacupuncture belong to acupuncture. In the realm of traditional Chinese medicine, the human body is a whole organism. Though the mechanism of CFS treated by acupuncture and moxibustion is not fully explained, the treatment is effective in relief of symptoms $[17,18]$. The acupuncture and moxibustion treatment can adjust organ function of traditional Chinese medicine, such as the zhang and fu, and have various ways in which they can be chosen depending on the characters of patients.

Even though some systematic review and metaanalysis were done before [19-21], there were also some limitations. The Alraek's study [21] didn't include acupuncture or moxibustion, therefore its relevance to this review is unclear, and the other two studies $[19,20]$ didn't conduct meta-analysis for acupuncture and moxibustion. Therefore comprehensive meta-analysis evidence is still not available that showed the therapeutic effect of comparing acupuncture and moxibustion with many others treatments. The network meta-analysis combines the comparisons of treatments not only directly addressed within any of the individual trials, but also incorporated the indirect comparisons constructed from two trials that share one treatment in two studies. This kind of meta-analysis makes full use of the withintrial randomized treatment comparison of each trial, while combining all available comparisons between treatments. This study attempts to evaluate the effect of acupuncture and moxibustion on CFS systematically compared with other treatments by traditional and network meta-analysis.

\section{Methods}

\section{Search strategy and study selection}

We searched through the databases of PubMed, EMBASE, Cochrane library, Chinese Biomedical Literature Database (CBM), China National Knowledge Infrastructure (CNKI), China Master Theses Full-text Database (CMTD), China Doctor/Maseter's Dissertations (CDMD) Fulltext Database. The last search for all databases was updated to May 2016. We used the combined method of MeSH Term and free words by applying the following terms: acupoint injection, acupuncture, acupuncture and moxibustion, acupuncture points, acupuncture therapy, auricular acupuncture, auricular plaster, body acupuncture, coiling dragon needling, dermal needle, dry needling, ear acupuncture, ear seed pressure, Electro-acupuncture, embedding, embedding therapy, fire needle, moxibustion, panlongci, percussopuncture, point injection, pricking blood, scalp acupuncture, trigger points, meridians and chronic fatigue syndrome, chronic fatigue, fatigue syndrome, Myalgic Encephalopathy in PubMed, EMBASE, Cochrane library and relevant Chinese words in CBM and CNKI.

\section{Inclusion and exclusion criteria Inclusion criteria}

(1) Randomized controlled trials (RCTs) in English or Chinese were included regardless of whether published or unpublished. (2) The patients were diagnosed under clear criteria (CDC 1988 [22] or CDC 1994 [2]); (3) RCT interventions adhered to the following treatment strategies: the experimental group received combined acupuncture plus moxibustion or acupuncture or moxibustion alone, while the control group received treatment with Chinese herbal medicine, western medicine, placebo treatment, acupuncture or moxibustion alone (for combined acupuncture plus moxibustion). (3) Clinical 
efficacy (cured, markedly effective, and effective) and invalid evaluation were used as the end-point [23], and the criteria for invalid evaluation was the syndrome score was reduced by less than $30 \%$ or $1 / 3$ [23].

\section{Exclusion criteria}

The exclusion criteria included the following: (1) nonRCTs or duplicate publications; (2) animal studies; (3) case reports and reviews; (4) clinical research studies that compared different kinds of acupuncture or moxibustion; (5) the treatment was combined with others treatments than acupuncture and moxibustion; (6) the study with western medicine which were clearly not recommended according to the latest NICE guideline, such as glucocorticoids, mineralocorticoids (https://www.nice.org.uk/guidance/cg53/chapter/ 1-Guidance\#genera-management-strategies-after-diagnosis); (7) the criteria of invalid evaluation was not based on syndrome score reduction by less than $30 \%$ or $1 / 3$.

\section{Quality assessment}

The quality assessment of all studies included in this review was independently evaluated by two reviewers (Wang TW and Xu C) using the Cochrane Collaborations tool [24]. Seven criteria were applied: (1) random sequence generation, (2) allocation concealment, (3) blinding of participants and personnel, (4) blinding of outcome assessment, (5) incomplete outcome data, (6) selective reporting and (7) other bias (defined as baseline data comparability). For each item, the evaluation was denoted as low, high or unclear risk according to the descriptions of the method in each study. Any disagreement was resolved by discussion with the third author (Xiong HY).

\section{Data extraction and analysis}

Related data such as title, first author, year of publishing, study design, intervention of each group, quality of the study, diagnosis criteria, number of participants, range of participants' age, outcomes, treatment duration and adverse events were independently extracted by two reviewers (Wang TW and $\mathrm{Xu} C$ ) using inclusion criteria. Disagreements were resolved by discussion between the two reviewers and by seeking the opinion of the third author (Xiong HY) if necessary.

Traditional meta-analysis was adopted for direct comparison. Random effects model was adopted for overall and subgroup analysis if obvious heterogeneity existed, otherwise fixed effects model. Furthermore, both models were adopted to test the difference of the two models for sensitivity analysis. At the same time, sensitivity analysis was finished by removing any single trial in each group. Statistical heterogeneity was evaluated by the Cochran's Chi-squared test (with $P<0.10$ indicating statistically significant heterogeneity) and the statistic $I^{2}$ [25] (The heterogeneity might not be important with $I^{2}$ of 0 to $40 \%$, while moderate heterogeneity with $I^{2}$ of 30 to $60 \%$, substantial heterogeneity with $I^{2}$ of 50 to $90 \%$ and considerable heterogeneity with $I^{2}$ of 75 to $100 \%$.). Publication bias was assessed by funnel plot and Egger's test (Egger's test was done only if the studies number was no less than 10) [25], whereas trim and fill [26] was performed if publication bias existed. To combine indirect and direct evidence, network meta-analysis was performed to evaluate the treatment effect of all treatments. We also compared the results of traditional meta-analysis and network meta-analysis. Effect sizes were calculated by related risk (RR) and 95\% confidence intervals (CIs). Meta-analysis was performed in $\mathrm{R}$ software version 3.2.0 [27] (with the package "meta" [28] for traditional meta-analysis and "pcnetmeta" [29] for Bayesian network meta-analysis).

\section{Results}

\section{Searching result}

A total of 1345 potentially relevant citations were identified, 47 of which were degree research thesis. 157 duplicate papers were removed firstly, and 1000 papers were excluded after scanning their titles and abstracts. After screening the full texts of the included articles, 158 studies were excluded for the following reasons: no relevant data $(n=35)$, inapposite treatments set $(n=73)$, patients with tuberculosis $(n=1)$, duplicate reports $(n=12)$, not RCT $(n=3)$, unclear diagnose criteria $(n=7)$, inapposite criteria of invalid evaluation $(n=24)$, inappropriate western medicine $(n=3)$. However, one additional article

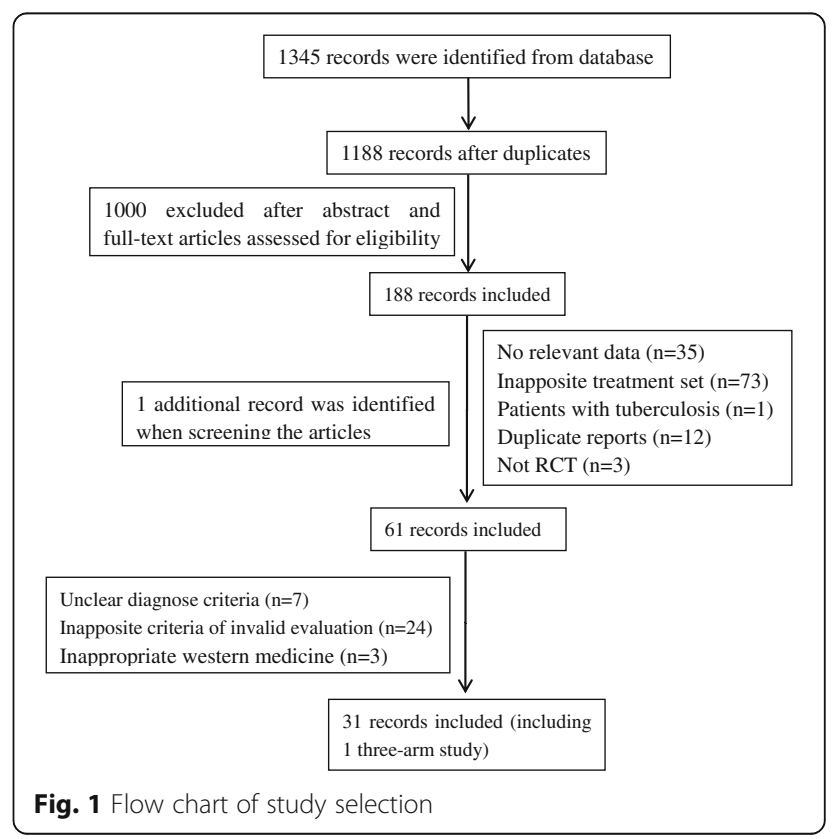




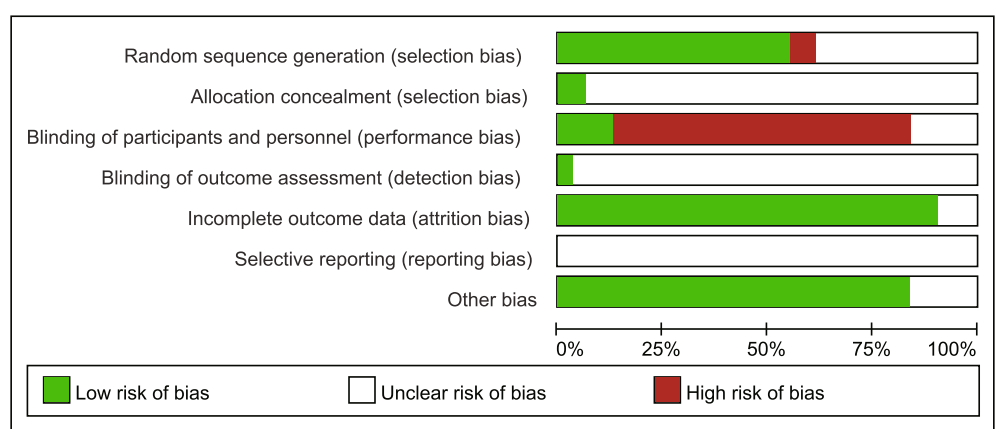

Fig. 2 The Cochrane risk of bias

[30] was identified during screening. Finally, 31 studies [17, 18, 30-58] (including1 three-arm study [18]) were included for further analysis (Fig. 1).

\section{Studies description}

We included 31studies (Additional file 1: Table S1), all of these were conducted in China and published in Chinese. The sample size of these studies ranged from 30 to 200, and the total number of patients was 2255 . The age of patients ranged from 18 to 78 , and the duration of treatment was from 10 to 90 days. All studies adopted the diagnosis criteria of American CDC (1988 or 1994 edition). As acupuncture and moxibustion had many forms, such as auricular acupuncture, electroacupuncture, which were acupuncture, we treated them all as acupuncturein the present study. Therefore, the control treatments were Chinese herbal medicine, western medicine, sham-acupuncture and SAM (single acupuncture or single moxibustion).

All the included studies were divided into five groups based on the intervention in experiment and control groups: (1) CbAM (combined acupuncture and moxibustion, which meant two or more types of acupuncture and moxibustion were adopted) versus Chinese herbal medicine $(n=8)$; (2) SAM versus Chinese herbal medicine $(n=12)$; (3) SAM versus western medicine $(n=4)$; (4) SAM versus sham-acupuncture (or placebo treatment) $(n=4)$; (5) CbAM versus SAM $(n=5)$.

\section{Quality of the included studies}

After assessing the quality of studies based on the basis of Cochrane risk of bias, we revealed that 17 trials reported random sequence generation $[17,18,31,33-36$, $38,39,42,44,45,49,51,53,54,58], 2$ studies provided information on allocation concealment $[17,51], 4$ trials described blinding of participants (single or double) [34, 39, 46, 49],1 trial described blinding of outcome assessment [39],3 trials had unclear bias of complete data $[17,33,58]$ and 5 trials had unclear other biases (defined as baseline data comparability) [34, 39, 45, 52, 55]. Selective reporting bias was unclear (Fig. 2).

\section{Synthesis of results}

\section{CbAM versus Chinese herbal medicine}

Eight trials [18, 35, 36, 40, 42, 50, 52, 56], 515 participants (259 in experiment group and 256 in control) were included in this group. The pooled results of the 8 trials that compared CbAM with Chinese herbal medicine were of RR (95\% CI), 1.17 (1.09-1.25), indicating that $\mathrm{CbAM}$ was more effective than Chinese

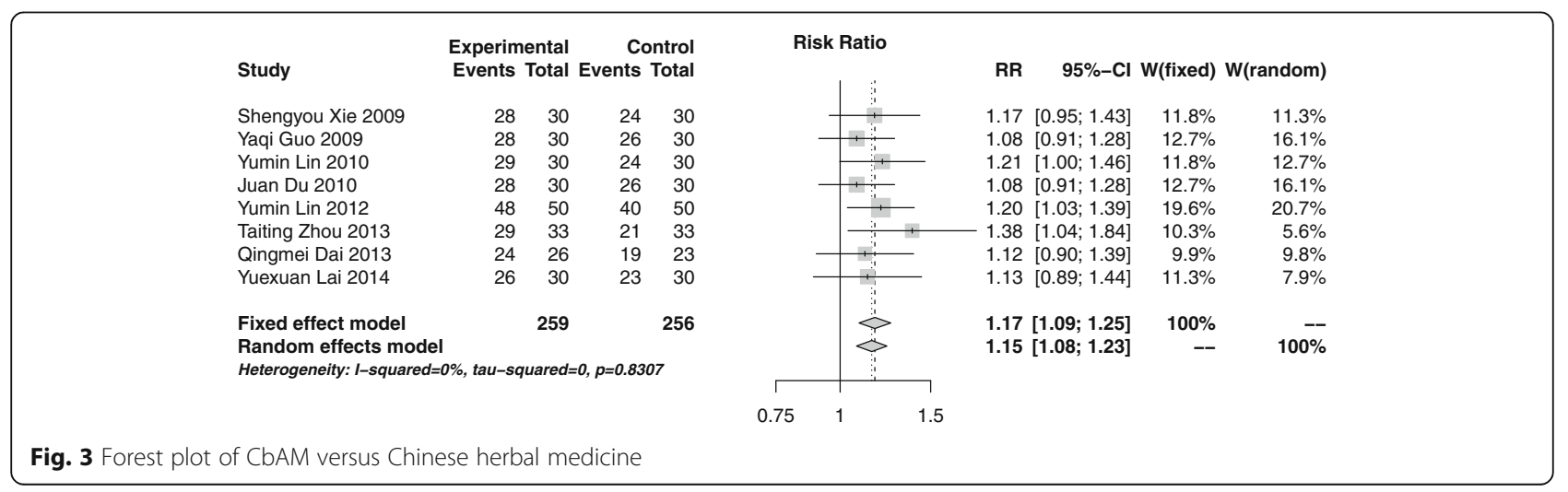




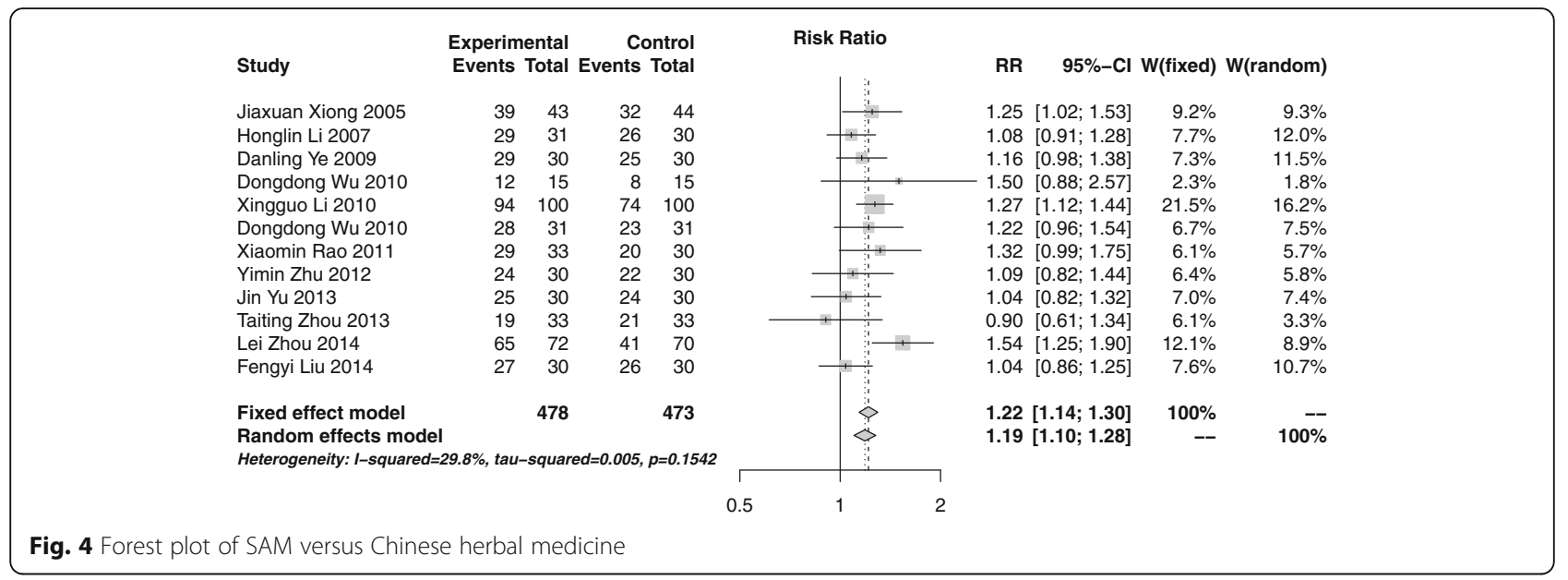

herbal medicine (Fig. 3). There were no heterogeneity $\left(I^{2}=0 \%, p=0.83\right)$.

\section{SAM versus Chinese herbal medicine}

Twelve trials [17, 18, 31, 33, 38, 41, 44, 45, 48, 51, 54, 58], 951 participants (478 in experiment group and 473 in control) were included in this group. With only not important heterogeneity existed $\left(I^{2}=29.8 \%, p=0.15\right)$, the pooled result of the 8 trials was performed with fixed effect model and the result (RR (95\% CI), 1.22 (1.14-1.30) showed that SAM had better effects than Chinese herbal medicine (Fig. 4). There was no publication bias after egger's test $(p=0.82)$.

\section{SAM versus western medicine}

A total of 4 trials [32, 37, 47, 53] (286 participants, 142 in experiment group and 144 in control) were adopted in this group. The pooled results indicated that SAM had a better effect than western medicine (RR (95\% CI), $1.51(1.31-1.74))$, with no heterogeneity among the studies $\left(I^{2}=0 \%, p=0.59\right)$ (Fig. 5).

\section{SAM versus placebo treatment (sham-acupuncture)}

Four trials [34, 39, 46, 49] (246 participants, 124 in experiment group and 122 in control) were conducted to compare SAM with sham-acupuncture. The pooled result indicated that SAM was much more effective than sham-acupuncture (RR (95\% CI), 5.90 (3.64-9.56)), with no heterogeneity $\left(I^{2}=0 \%, p=0.45\right)$ (Fig. 6).

\section{CbAM versus SAM}

A total of 5 trials [18, 30, 43, 56, 57], 356 participants (178 in experiment group and 178 in control) were involved in this group. The pooled results revealed that CbAM was more effective than SAM (RR (95\% CI), 1.23 $(1.12-1.36)$ ) (Fig. 7). The statistic $I^{2}$ for homogeneity of RR showed there was no heterogeneity among the studies $\left(I^{2}=13.1 \%, p=0.33\right)$.

Sensitivity analyses were performed in all traditional meta-analysis. No small sample effect existed after removing any single trial. Random effect model was also adopted and the results were similar to the fixed random effect, showing stable results (Figs. 3, 4, 5, 6 and 7).

\section{Network meta-analysis}

The results of network meta-analysis were similar with the traditional meta-analysis (Table 1), showed that all treatments were more effective than sham-acupuncture, and the comprehensive rank (from high to low effective

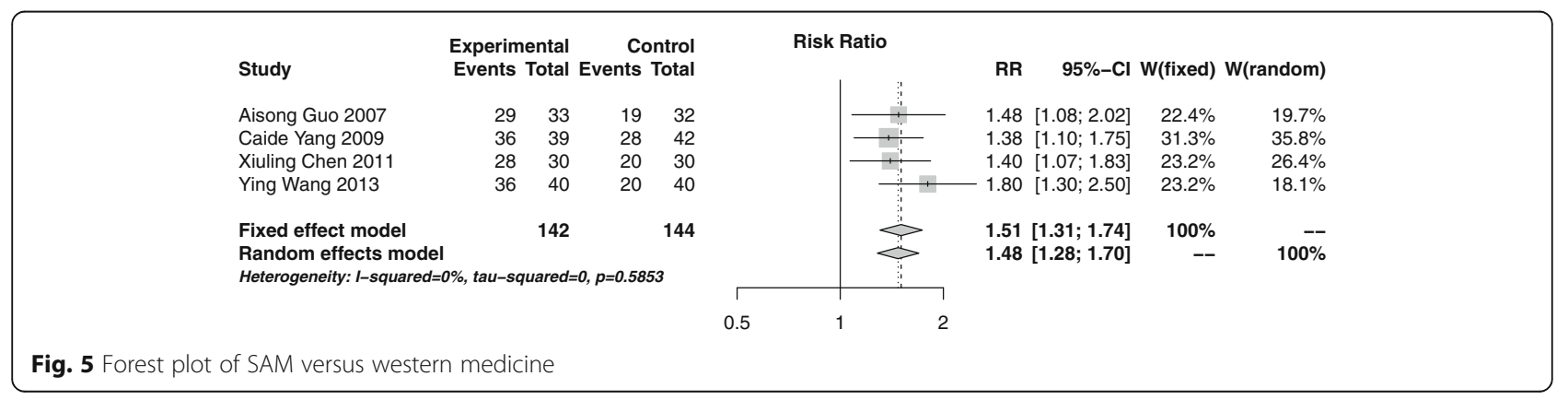




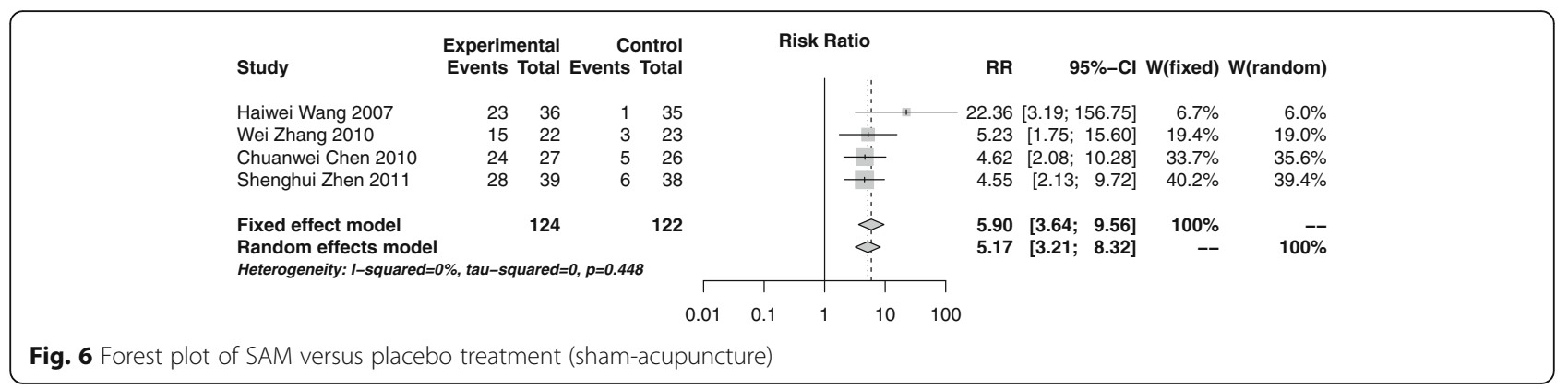

rate) was CbAM, SAM, Chinese herbal medicine, western medicine and sham-acupuncture (Figs. 8 and 9)

\section{Adverse events}

Of the conducted trials, adverse events happened in four studies [17, 31, 33, 42]. In total, there was fainting during acupuncture (6 cases), feeling of acid bilges on the back (6 cases),subcutaneous hematoma (1 case), burn during moxibustion (1 case). No serious adverse events happened.

\section{Acupuncture point}

The acupoints were chosen with two ways, constant point and constant point plus points based on the basis of symptoms. After analysis of points adopted in trial, we found Walking Three Miles (ST36), Spleen Locus (BL20), and Liver Locus (BL18) were the three points most commonly used (Additional file 2: Figure S1).

\section{Discussion}

In our meta-analysis, we included 31 trials for traditional and network meta-analysis. In traditional meta-analysis, CbAM and SAM were more effective than Chinese herbal medicine, western medicine and sham-acupuncture. In network meta-analysis, similar results were found. We also analysed all treatments by network meta-analysis, of which CbAM was the most effective, followed by SAM, Chinese herbal medicine, western medicine, and then sham-acupuncture.
The comparison of network meta-analysis and traditional meta-analysis depicted the same qualitative results (Table 1). It is reported that network metaanalysis can get narrower $\mathrm{CI}$ for which it can combine direct and indirect results [59]. In this research, similar conclusions were observed in comparison of CbAM versus SAM. However, in the comparison of SAM versus western medicine, SAM versus sham-acupuncture, the $95 \%$ CI became broader in network meta-analysis. This may be due to the inconsistency between direct and indirect comparison. The inconsistency test also showed that inconsistency existed in triangular loop of CbAM, SAM, Chinese herbal medicine, suggesting that the quantitative results of comparison needed further research although the same qualitative results were obtained.

Even though more and more evidences show the efficiency of acupuncture and moxibustion, there is still doubt that the functions of acupuncture and moxibustion are owing to placebo effect $[60,61]$. In our investigation we confirmed the efficiency of acupuncture and moxibustion by traditional and network meta-analysis, showing acupuncture and moxibustion had better effects than the placebo and also Chinese herbal medicine, western medicine, and similar results of acupuncture and moxibustion were also obtained in treating chronic pain when compared with placebo treatment [62]. Although the mechanism of acupuncture and moxibustion is still unclear, and some evidences of eradicating diseases and keeping healthy by means of acupuncture

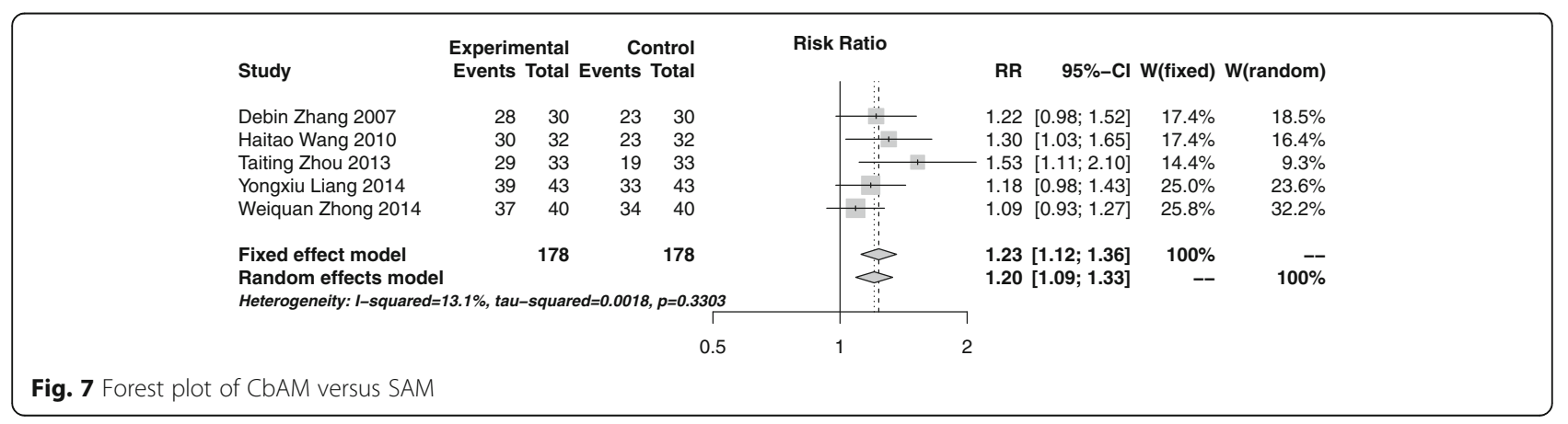




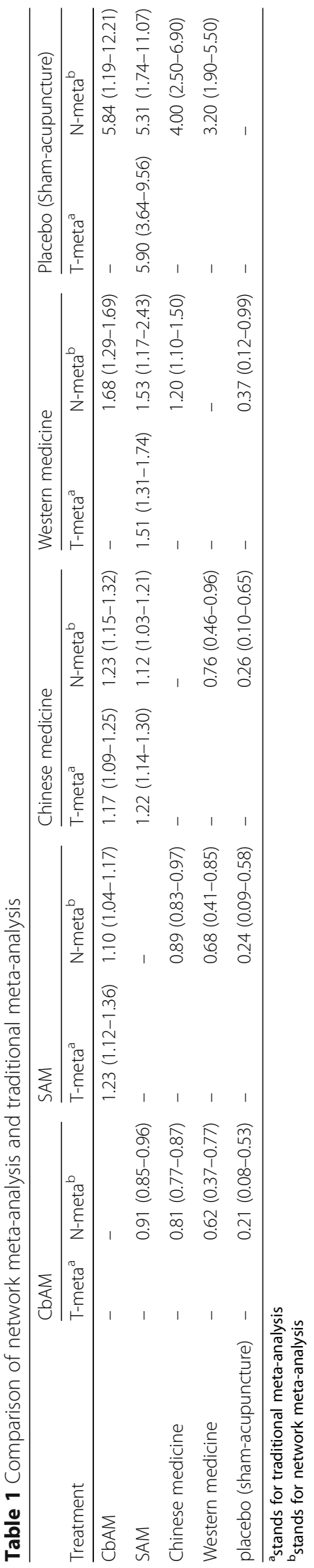




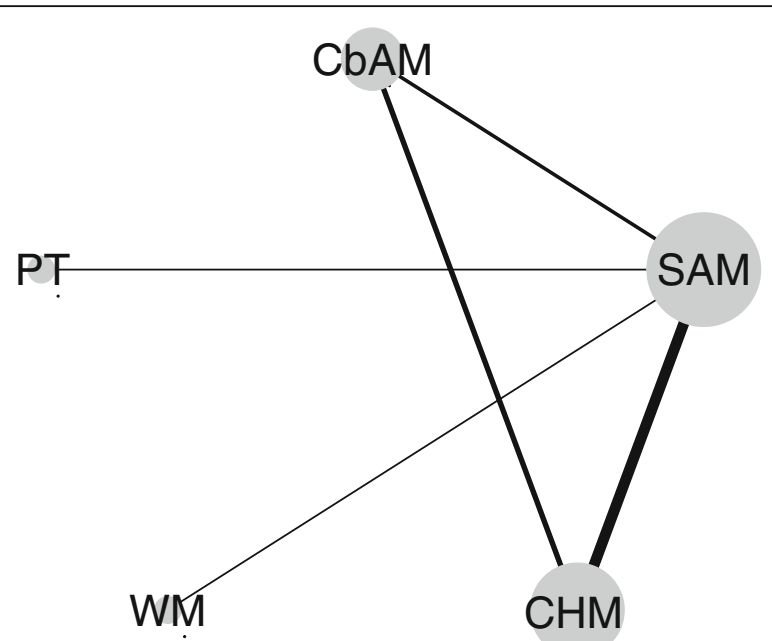

Fig. 8 Network of involved treatments. CbAM, combined acupuncture and moxibustion; SAM, single acupuncture or single moxibustion; CHM, Chinese herbal medicine; WM, western medicine; PT, placebo treatment (sham-acupuncture)

and moxibustion from western medicine have been observed. In the trials of Zhen [63], Xiong [64], they found that immunity factors IL- 6 , IFN- $\gamma$, TNF- $\alpha$ changed significantly after the treatment with acupuncture and moxibustion, indicating that the treatment could strengthen the immune system. In animal experiments $[65,66]$, compared with normal rats, the CFS rats had lower immunity factors of IgA, IgM, IgG and IFN- $\gamma$. After the treatment of acupuncture and moxibustion, the immunity factors recovered again indicating CFS might be caused by immune function disorder, and regulating immunity may be one of the methods that acupuncture and moxibustion adopt to treat CFS.
In this research, we focused on quantitative assessment of acupuncture and moxibustion by traditional and network meta-analysis, and compared it with Chinese herbal medicine and western medicine. Even though some similar researches have been performed before [19-21], some new informations were provided, for example, network meta-analysis. We also analyzed the acupoints adopted in trials, which was found to be a little different with the previous research [19]. The head five acupoints were Walking Three Miles (ST36), Crossroad of Three Yins (SP6), Hundred Meetings (GV20), Back Area of Governing Vessel Meridian, Back Area of Urinary Bladder Meridian [19] and Walking Three Miles (ST36), Spleen Locus (BL20), Liver Locus (BL18), Kidney Locus (BL23), Crossroad of Three Yins (SP6) in our research. The reasons of the difference may be the criteria of inclusions and exclusions, and that the research time was different, we enrolled more trials than the earlier research. The acupoints information could provide some suggestions when chronic fatigue syndrome patients were treated by acupuncture and moxibustion.

From our results, the traditional Chinese medicine, as a type of complementary and alternative medicine, has good effects in relief of CFS symptoms. However, the others complementary and alternative medicines need further exploration. Furthermore, a group-based selfmanagement program for chronic fatigue syndrome patients seems to be useful in relief of fatigue severity, despite unsustained at the one-year follow-up, [67] therefore, it is needed to support CFS/ME patients to access reliable, evidence-based information outside primary care, and an online resource for patients to support self-management may be a good choice [68]. In addition, activity pacing self-management was also found to be feasible and effective in desired daily life activities and reducing fatigue in women with CFS [69].
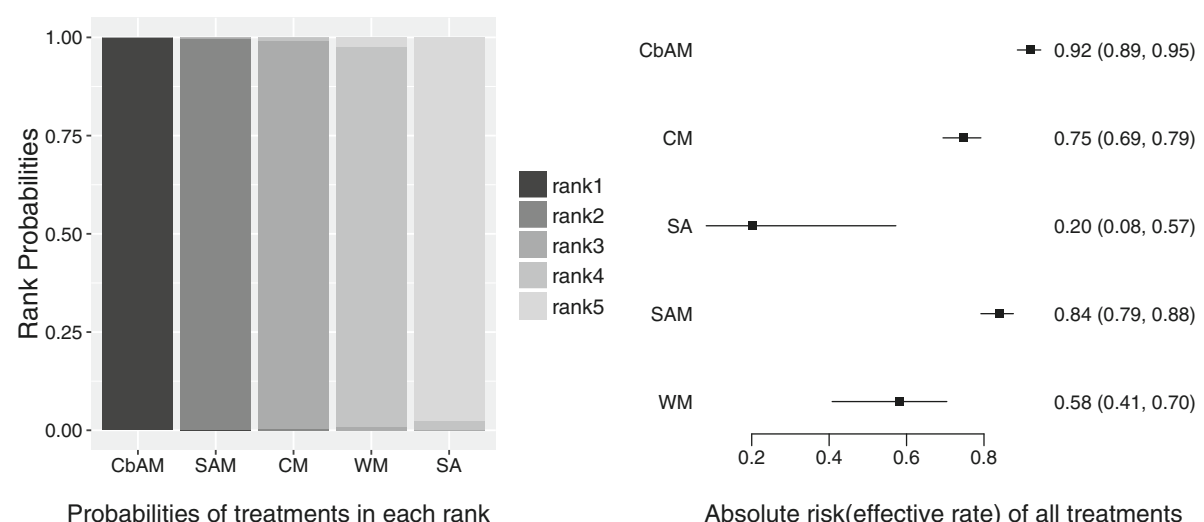

Absolute risk(effective rate) of all treatments

Fig. 9 Comprehensive comparison of all treatments. Left, probabilities of all treatments in each rank; right, absolute risk (effective rate) of all treatments 
In this meta-analysis, we concentrated on the effect of acupuncture and moxibustion, and did not include trials for Chinese herbal medicine versus western medicine. Through network meta-analysis, we found Chinese herbal medicine had better effect than western medicine, which was similar to a traditional meta-analysis of Chinese herbal medicine to western medicine [70], and these two similar results provided extra evidence that our results were relatively reliable.

\section{Limitations}

In this review, 31 RCTs for treating CFs were identified, but the quality wasn't high. Some factors that could influence the results were discussed below. First, the quality of methodology of the studies was poor. Only two studies provided information on allocation concealment [17, 51], four trials described blinding of participants (single or double) [34, 39, 46, 49], one trial described blinding of outcome assessment [39], and three trials had unclear bias of complete data $[17,33,58]$. These characteristics may lead to bias in selection, performance, and detection and may result in false-positive findings. However, this is a more comprehensive result so far. Second, as the limitation of sample size in some groups, such as SAM versus western medicine, some results still need further confirmations. Third, the qualitative results of some comparisons between traditional and network metaanalysis were a little different, indicating that the exact effects need further exploring. Fourth, there was potential publication bias among the included studies. All these trials were conducted in China and published in Chinese. Therefore positive results may be easier to be published. However, the heterogeneity might not be important as the $I^{2}$ in all groups which was below 30\%, that showed good effects of acupuncture and moxibustion. Fifth, although the outcome measure of effective rate was widely used in many related studies. The outcome measure was really a limitation due to the reason that effective rate was not a validated outcome and the definition could be subjective. This further limits the reliability of the findings.

\section{Conclusions}

In this systematic review, we evaluated the treatment effect of acupuncture and moxibustion comprehensively which were found to be more effective than Chinese herbal medicine, western medicine and placebo treatment (sham-acupuncture) in relieving symptoms. However, because of low quality evidence and heterogeneity, further studies are required to confirm this hypothesis.

\section{Additional files}

Additional file 1: Table S1. The characteristics of included studies. (PDF 630 kb)

Additional file 2: Figure S1. The highest frequency acupoints adopted in studies. (PDF $123 \mathrm{~kb}$ )

Abbreviations

AM: Acupuncture and moxibustion; CFS: Chronic fatigue syndrome; TCM: Traditional Chinese medicine

Acknowledgements

We thank Chongaing Boyu Foreign Language Translation Center for assisting in the preparation of this manuscript.

\section{Funding}

This work was supported by a grant from the Special Health Research Project, Ministry of Health of China (No. 201002012) and a grant from Chongqing Health and Family Planning Commission (No. 20141027).

\section{Availability of data and materials}

The datasets supporting the conclusions of this article are included within the article.

\section{Authors' contributions}

WTW conducted the database search, assessed studies for inclusion, extracted and analyzed the data, and prepared the manuscript draft. CX assessed studies for inclusion, extracted the data which followed by cross checking with WTW. KLP analyzed the data and prepared the manuscript draft with WTW. HYX conceived the study and revised the manuscript. All authors read and approved the final version of the manuscript.

\section{Competing interests}

The authors declare that they have no competing interests.

Consent for publication

Not applicable.

Ethics approval and consent to participate

Not applicable.

Received: 25 June 2016 Accepted: 23 February 2017

Published online: 23 March 2017

\section{References}

1. Jason LA, Richman JA, Rademaker AW, Jordan KM, Plioplys AV, Taylor RR, et al. A community-based study of chronic fatigue syndrome. Arch Intern Med. 1999;159(18):2129-37.

2. Fukuda K, Straus SE, Hickie I, Sharpe MC, Dobbins JG, Komaroff A. The chronic fatigue syndrome: a comprehensive approach to its definition and study. International Chronic Fatigue Syndrome Study Group. Ann Intern Med. 1994;121(12):953-9.

3. Lindal E, Stefansson JG, Bergmann S. The prevalence of chronic fatigue syndrome in Iceland-a national comparison by gender drawing on four different criteria. Nord J Psychiatry. 2006;60(2):183.

4. Ranjith G. Epidemiology of chronic fatigue syndrome. Occup Med (Lond). 2005:55(1):13-9.

5. Rimes KA, Goodman R, Hotopf M, Wessely S, Meltzer H, Chalder T. Incidence, prognosis, and risk factors for fatigue and chronic fatigue syndrome in adolescents: a prospective community study. Pediatrics. 2007:119(3):e603-9.

6. Lee S, Yu H, Wing Y, Chan C, Lee AM, Lee DT, et al. Psychiatric morbidity and illness experience of primary care patients with chronic fatigue in Hong Kong. Am J Psychiatry. 2000;157(3):380-4.

7. Burgess M, Andiappan M, Chalder T. Cognitive behaviour therapy for chronic fatigue syndrome in adults: face to face versus telephone treatment: a randomized controlled trial. Behav Cogn Psychother. 2012;40(2):175-91.

8. Price JR, Mitchell E, Tidy E, Hunot V. Cognitive behaviour therapy for chronic fatigue syndrome in adults. Cochrane Database Syst Rev. 2008;3:CD001027. 
9. Malouff JM, Thorsteinsson EB, Rooke SE, Bhullar N, Schutte NS. Efficacy of cognitive behavioral therapy for chronic fatigue syndrome: a meta-analysis. Clin Psychol Rev. 2008;28(5):736-45.

10. Larun L, Brurberg KG, Odgaard-Jensen J, Price JR. Exercise therapy for chronic fatigue syndrome. Cochrane Database Syst Rev. 2016;6:CD003200.

11. Chambers D, Bagnall AM, Hempel S, Forbes $C$. Interventions for the treatment, management and rehabilitation of patients with chronic fatigue syndrome/myalgic encephalomyelitis: an updated systematic review. J R Soc Med. 2006;99(10):506-20.

12. Reid S, Chalder T, Cleare A, Hotopf M, Wessely S. Chronic fatigue syndrome. BMJ Clin Evid. 2011;05:1-56.

13. Carruthers B, Jain A, De Meirleir K, Peterson D, Klimas N, Lerner A. Myalgic encephalomyelitis/chronic fatigue syndrome: clinical working case definition, diagnostic and treatment protocols. J Chronic Fatigue Syndr. 2003;11(7):115.

14. Turnbull N, Shaw EJ, Baker R, Dunsdon S, Costin N, Britton G, et al. Chronic fatigue syndrome/myalgic encephalomyelitis (or encephalopathy): diagnosisand management of chronic fatigue syndrome/ myalgicencephalomyelitis (or encephalopathy) in adults and children. London: Royal College of General Practitioners; 2007.

15. Afari N, Buchwald D. Chronic fatigue syndrome: a review. Am J Psychiatry. 2003:160(2):221-36.

16. Alecrim-Andrade J, Maciel-Junior JA, Cladellas XC, Correa-Filho HR, Machado HC. Acupuncture in migraine prophylaxis: a randomized sham-controlled trial. Cephalalgia. 2006;26(5):520-9.

17. Liu FY. The study of Thread Embedding Therapy with Shu Mu Combination by in Treatment of Liver Depression and Spleen Deficiency of Chronic Fatigue Syndrome. Guangzhou University of Chinese Medicine Master's Thesis. 2014.

18. Zhou TT. The clinical effects and immune mechanism research of moxibustion with warming needle for chronic fatigue syndrome with deficiency of heart and spleen. Guangzhou University of Chinese Medicine Doctor's Thesis. 2013.

19. Wang $T$, Zhang $Q$, Xue $X$, Yeung A. A systematic review of acupuncture and moxibustion treatment for chronic fatigue syndrome in China. Am J Chin Med. 2008;36(1):1-24.

20. Wang YY, Li XX, Liu JP, Luo H, Ma LX, Alraek T. Traditional Chinese medicine for chronic fatigue syndrome: a systematic review of randomized clinical trials. Complement Ther Med. 2014;22(4):826-33.

21. Alraek T, Lee MS, Choi TY, Cao H, Liu J. Complementary and alternative medicine for patients with chronic fatigue syndrome: a systematic review. BMC Complement Altern Med. 2011:11:87.

22. Holmes GP, Kaplan JE, Gantz NM, Komaroff AL, Schonberger LB, Straus SE, et al. Chronic fatigue syndrome: a working case definition. Ann Intern Med. 1988;108(3):387-9.

23. Liu Q. The curative effect standard of TCM diagnosis and treatment of chronic fatigue syndrome. Foreign Med Fascicle Chin Med. 1993;15(6):15.

24. Higgins JP, Altman DG, Gotzsche PC, Juni P, Moher D, Oxman AD, et al. The Cochrane Collaboration's tool for assessing risk of bias in randomised trials. BMJ. 2011;343:d5928.

25. Egger M, Davey Smith G, Schneider M, Minder C. Bias in meta-analysis detected by a simple, graphical test. BMJ. 1997;315(7109):629-34.

26. Duval S, Tweedie R. Trim and fill: a simple funnel-plot-based method of testing and adjusting for publication bias in meta-analysis. Biometrics. 2000;56(2):455-63.

27. R. Core T. R: A language and environment for statistical computing. Vienna: R Foundation for Statistical Computing; 2015. http://www.R-project.org/.

28. Schwarzer G. meta:General Package for Meta-Analysis. Version: 4.1-0. http:// cran.r-project.org/web/packages/meta/index.html. [Accessed 25 Feb 2017].

29. Lin LF, Zhang J, Chu HT. pcnetmeta: Patient-Centered Network MetaAnalysis Version: 2.1. http://cran.r-project.org/web/packages/pcnetmeta/ index.html. [Accessed 25 Feb 2017]

30. Zhang DB, Zhou CX, Liu L. Clinical observation of chronic fatigue syndrome treated by warm acupuncture and moxibustion at jiaji. Chin Acupunct Moxibustion. 2007;51:61-62.

31. Xiong JX. The clinical researches of electro-therapy treatment for chronic fatigue syndromes. Guangzhou University of Traditional Chinese Medicine Master's Thesis. 2005.

32. Guo AS, Gu YH, Jin HZ. Clinical comparative study of moxibustion on chronic fatique syndrome. J Liaoning Univ Tradit Chin Med. 2007;9(4):29-30.

33. Li HL. Clinical research on CFS of "Ganyupixu" kind with the SHU-MU network points acupuncture. Heilongjiang University of Chinese Medicine Master's Thesis. 2007.
34. Wang HW. Clinical study on electroacupuncture treatment of chronic fatigue syndrome. Chengdu university of TCM Doctor's Thesis. 2007.

35. Guo YQ. The clinical study of combined therapy of body and auricular acupuncture in the treatment of chronic fatigue syndrome. Guangzhou University of Chinese Medicine Doctor's Thesis. 2009.

36. Xie SY. The clinical study of combined therapy of electro- and auricular acupuncture in the treatment of chronic fatigue syndrome. Guangzhou University of Chinese Medicine Master's Thesis. 2009.

37. Yang CD, Bao JL, Song JC, Qiu YY. Clinical observation of 80 CFS cases treated with catgut embedment in acupoints. World Chin Med. 2009;4(3):154-5.

38. Ye DN. The effective observation of curing liver depression and spleen deficiency of chronic fatigue syndrome by acupuncture Guangzhou University of Chinese Medicine Master's Thesis. 2009.

39. Chen CW. Mechanism and clinical observation on acupuncture in chronic fatigue syndrome. Guangzhou University of Chinese Medicine Doctor's Thesis. 2010.

40. Du J. Clinical study on treatment of chronic fatigue syndrome of stagnation of the liver-qi and spleen-deficiency with acupuncture and moxibustion. Changchun University of Chinese Medicine Master's Thesis. 2010.

41. Li XG. Clinical research on treating chronic fatigue syndrome by elongated needle penetration needling plus holistic nursing. Clin J Chin Med. 2010;2(20):18-9.

42. Lin YM. The clinical researches of moxibustion with warming needle for chronic fatigue syndrome. Guangzhou University of Chinese Medicine Master's Thesis. 2010.

43. Wang HT. Observation on curative effect of warm acupuncture and moxibustion at back - shu points on chronic fatigue syndrome. Chinical J Clin Ration Drug Use. 2010;3(17):78.

44. Wu DD. The clinical research of eight meridian confluence points with auricular therapytreatment of chronic fatigue syndrome of liver depression and spleen. Heilongjiang university of Chinese medicine Master's Thesis. 2010.

45. Wu DD, Li Y. The treatment of both heart and spleen deficiency type of chronic fatigue syndrome by eight confluent points with ear acupuncture. J Clin Acupunct Moxibustion. 2010;26(11):31-3.

46. Zhang W. Clinical study on acupuncture at Back-Shu points for chronic fatigue syndrome:a report of 22 cases. J Tradit Chin Med. 2010;51(2):139-41.

47. Chen XL, Xu K, Zhou J, Huang YS, Lin HG, Luo RH. Clinical observation on chronic fatigue syndrome treated by moxibustion at Guanyuan and Qihai. J New Chin Med. 2011;43(2):109-10.

48. Rao XM, Luo ZN, Wang XL. Treatment of 33 cases of chronic fatique syndrome by heat-sensitive moxibustion. Jiangxi J Tradit Chin Med. 2011:5(42):66-7.

49. Zhen $S H$, Zhen $S Z$, Jiao JK, Ren R, Wei LL, Yang LX, et al. Effect of acupuncture and moxibustion of Shu-Mu points on quality of life of patients with chronic fatigue syndrome. Guiding I Tradit Chin Med Pharm. 2011;17(7):66-8.

50. Lin YM, Chen WJ, Chen XL, Jiang GH. Therapeutic effect of moxibustion with warming needle for chronic fatigue syndrome with heart-spleen deficiency: an observation of 50 cases. J New Chin Med. 2012;44(10):93-4.

51. Zhu YM. Abdominal acupuncture for treatment of chronic fatigue syndrome a randomized clinical trial. Nanjing University of Chinese Medicine Doctor's Thesis. 2012.

52. Dai $\mathrm{QM}$, Chen HB. Treatment of 26 cases of chronic fatique syndrome with Yiqiwenyang and acupuncture. Nei Mongol J Tradit Chin Med. 2013;40:46.

53. Wang $Y$, Xiao W, Wang J. Therapeutic obsenvation on thunder-fire moxibustion for chronic fatigue syndrome. Shanghai J Acupunct Moxibustion. 2013;32(10):827-8.

54. Yu J. Clinical research on treatment of chronic fatique syndrome by acupuncture on back points. Dalian Medical University Master's Thesis. 2013.

55. Lai XY. The acupuncture treatment of Spirit selection points to the liver depression and spleen deficiency syndrome of chronic fatigue syndrome clinical observation. Nanjing University of Traditional Chinese Medicine Doctor's Thesis. 2014.

56. Liang YX, Liu Q. Observation on curative effect of acupoint catgut embedding with moxibustion of moxibustion on chronic fatigue syndrome. J Pract Tradit Chin Med. 2014;30(7):642-3.

57. Zhong WQ, Li SC, Gu TT. Observation on curative effect of warming acupuncture on chronic fatigue syndrome. Shanghai J Acu-mox. 2014;33(3):206-8.

58. Zhou L, Feng ZG. Efficacy of chronic fatigue syndrome treated by acupoint catgut-embedding. J Clin Acupunct Moxibustion. 2014;30(2):31-3.

59. Bangalore S, Kumar S, Kjeldsen SE, Makani H, Grossman E, Wetterslev J, et al. Antihypertensive drugs and risk of cancer: network meta-analyses and trial sequential analyses of 324,168 participants from randomised trials. Lancet Oncol. 2011;12(1):65-82. 
60. Linde K, Streng A, Jurgens S, Hoppe A, Brinkhaus B, Witt C, et al. Acupuncture for patients with migraine: a randomized controlled trial. JAMA. 2005;293(17):2118-25.

61. Diener HC, Kronfeld K, Boewing G, Lungenhausen M, Maier C, Molsberger A, et al. Efficacy of acupuncture for the prophylaxis of migraine: a multicentre randomised controlled clinical trial. Lancet Neurol. 2006;5(4):310-6.

62. Vickers AJ, Cronin AM, Maschino AC, Lewith G, MacPherson H, Foster NE, et al. Acupuncture for chronic pain: individual patient data meta-analysis. Arch Intern Med. 2012;172(19):1444-53.

63. Zhen SH, Zhen SZ, Jiao JK, Ren R, Wei L. Clinical efficacy of Back-Shu and Front-Mu point acupuncture on chronic fatigue syndrome and its effect on serum level of cytokines. Chin J Inf TCM. 2012;19(1):10-2.

64. Xiong S. The Change of IL-6,IFN- - , TNF- $a$ and other Cytokines of CFS Treating though Accupuncture and massage to bladder Meridian. Hubei University of Chinese Medicine Master's Thesis. 2013

65. Cheng ZD, Zhao Y, Chen YG. Influence of mild moxibustion at Guanyuan point on immunoglobulins of chronic fatigue rats. Chin Arch Tradit Chin Med. 2013;31(2):339-41.

66. Tang $C Z$, Yang $L X$, Yang $L$, Xia JX, Xian ZH. Influence of acupuncture on serum IFN- $\gamma$ and TGF- $\beta$ in rats with chronic fatigue syndrome. Chin J Basic Med Tradit Chin Med. 2013;13(8):613.

67. Pinxsterhuis I, Sandvik L, Strand EB, Bautz-Holter E, Sveen U. Effectiveness of a group-based self-management program for people with chronic fatigue syndrome: a randomized controlled trial. Clin Rehabil. 2015;31(1):93-103.

68. Bayliss K, Riste L, Band R, Peters S, Wearden A, Lovell K, et al. Implementing resources to support the diagnosis and management of Chronic Fatigue Syndrome/Myalgic Encephalomyelitis (CFS/ME) in primary care: a qualitative study. BMC Fam Pract. 2016;17:66

69. Kos D, van Eupen I, Meirte J, Van Cauwenbergh D, Moorkens G, Meeus M, et al. Activity pacing self-management in chronic fatigue syndrome: a randomized controlled trial. Am J Occup Ther. 2015;69(5):6905290020.

70. Peng W, Su J, Xu Q, Wang QJ, Jiang XJ. A meta-analysis of chronic fatigue syndrome treated by Chinese medicine. Chin J GuangMing Chin Med. 2013;28:1345-9

\section{Submit your next manuscript to BioMed Central and we will help you at every step:}

- We accept pre-submission inquiries

- Our selector tool helps you to find the most relevant journal

- We provide round the clock customer support

- Convenient online submission

- Thorough peer review

- Inclusion in PubMed and all major indexing services

- Maximum visibility for your research

Submit your manuscript at www.biomedcentral.com/submit

) Biomed Central 